ISSN e- 2594-1100

en-claves del pensamiento / núm. 30 / julio-diciembre / 2021 / e434

https://doi.org/10.46530/ecdp.v0i30.434 ARTÍCULOS

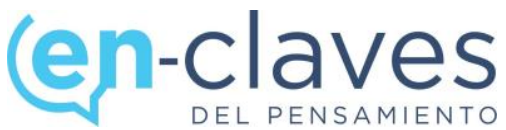

\title{
GUERRA GLOBAL CONTRA EL TERRORISMO: OCCIDENTALIZACIÓN FALLIDA
}

\author{
Global War on Terror (GWOT): Failed Westernization
}

Jacobo Salvador Micó Faus, Action and Communication on the Middle East-com.es, España

Correo electrónico: jacobo2388@gmail.com

Recibido: $24 / 09 / 2020$

Aceptado: $10 / 11 / 2020$

Publicado: 01/07/2021

\begin{abstract}
Resumen. Con base en la definición de guerra, según expertos en polemología y el estudio de las guerras 'justas' e 'injustas' y de acuerdo con los autores más relevantes en la doctrina filosófica de guerra, la Guerra Global contra el Terrorismo (GWOT, por sus siglas en inglés) no es una guerra como tal. El hecho de aplicar tal conceptualización a una serie de acontecimientos donde lo que se combate es el fenómeno terrorista carece de sentido desde el momento que el terrorismo no es más que un arma de guerra. Además, la injusticia de tales actos le restaría valor al concepto de guerra 'justa' defendido por los autores clásicos. Si se acepta que la GWOT no es una guerra como tal, nos preguntamos qué es, entonces: ¿una respuesta indirectamente buscada?; ¿un intento de democratización balístico?; ¿o la continuación de los conflictos civilizacionales tras la Guerra Fría? Sea lo que sea, la inestabilidad en Medio Oriente y el terrorismo han aumentado.
\end{abstract}

Palabras claves: guerra, terrorismo, EE. UU, occidentalización, guerra injusta.

\begin{abstract}
Based on the definition of war, according to experts in polemology and the study of 'just' and 'unjust' wars and according to the most relevant authors in the philosophical doctrine of war, the Global War on Terrorism (GWOT) is not a war as such. Applying such a conceptualization to a series of events where what is being combated is the terrorist phenomenon makes no sense since terrorism is nothing more than a weapon of war. Moreover, the injustice of such acts would detract from the concept of 'just' war defended by the classical authors. If it is accepted that the GWOT is not a war as such, we wonder what it is, then: An indirectly sought answer? An attempt at ballistic democratization? Or the continuation of civilizational conflicts after the Cold War? Whatever the answer, instability in the Middle East and terrorism have increased.
\end{abstract}

Keywords: War, terrorism, USA, Westernization, unjust war.

Micó Faus, J. (2021). Guerra Contra el Terrorismo (GWOT) — Occidentalización fallida. EN-CLAVES del pensamiento, O(30), e434. doi: https://doi.org/10.46530/ecdp.v0i30.434

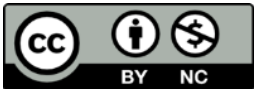

Esta obra está protegida bajo una Licencia

Creative Commons Atribución - No comercial

4.0 Internacional

www.enclavesdelpensamiento.mx

https://doi.org/10.46530/ecdp.v0i30.434 
Si existiera algo que quisiéramos cambiar, en primer lugar, deberíamos examinarlo y observar si no es algo que podría ser mejor cambiar en nosotros mismos.

Carl Gustav Jung

Si queremos que continúe como está, es necesario que todo cambie. Giuseppe Tomasi di Lampedusa

Si eres tú quien provoca el cambio, ya no te asustará. Spencer Johnson

\section{Introducción}

La Guerra contra el Terrorismo o Global War on Terror $(\text { GWOT })^{1}$ fue fruto de una respuesta ineludible por parte de la administración estadounidense ante los atentados del 11 de septiembre de 2001. Los hechos supusieron una declaración de guerra a la primera potencia mundial a cuya respuesta se unieron un amplio número colaboradores, involucrándose así gran parte de la comunidad internacional. La campaña iniciada por Estados Unidos junto varios miembros de la OTAN y otros aliados ha tenido como objetivo acabar con el terrorismo internacional.

\section{La GWOT no es una guerra justa}

Existen dos argumentos de peso mediante los cuales podríamos afirmar por qué la guerra contra el terrorismo no es una "guerra justa" como tal. El primer de ellos lo encontramos en su propia definición, ya que los expertos en polemología rechazan la posibilidad de que tales actos encajen dentro de dicho concepto. Además, rechazan la idea de que una guerra pueda ser declarada frente al terrorismo ya que éste es un instrumento de guerra. La guerra es uno de los medios utilizados para solucionar conflictos por lo que emplearla para combatir el terrorismo carece de lógica. Si se me permite el símil, sería como pretender

${ }^{1}$ Cristóbal Pérez, "La Guerra Global contra el Terrorismo (GWOT)", Boletín de información, núm. 324 (2012), 7-48. 
destruir un diente de la hoja de una sierra utilizando la propia sierra, siendo el diente uno de los tantos elementos de los que se sirve.

La segunda premisa que permite negarla es su caracterización como guerra 'injusta'. Las guerras injustas carecen de utilidad debido a que su fin no es la paz, objetivo final de todas ellas si tomamos como referencia la definición que se da guerra justa en la filosofía clásica. Resulta importante matizar que nos referimos a una paz parcial y no a la extrapolación que Kant hace de 'paz perpetua' y su mundo ideal. ${ }^{2}$ La paz perpetua sólo sería posible con el fin de los tiempos, mientras que las guerras del presente adoptan una concepción de paz material con un sustrato en su capa basal donde las sociedades políticas y Estados se enfrentan para mantener sus intereses con la intención de volver a un estado de no conflicto. ${ }^{3}$ En ocasiones estos intereses no se ven satisfechos con paces parciales y en una fase posterior donde se da la oportunidad aparecen nuevas reivindicaciones, de tal modo los Estados pretenden así alcanzar un equilibrio estableciendo un continuum de “paces parciales". Tal concepción se aproxima a la connotación positiva de conflictos debido a la posibilidad que otorgan de enriquecer las relaciones sin necesidad de establecer vencedores ni vencidos. ${ }^{4}$

Siguiendo la tesis de Clausewitz, estos enfrentamientos derivan en una guerra en el momento que la diplomacia no consigue satisfacer sus intereses, por ello, las guerras del presente son un instrumento en manos de los Estados que les permite saciar sus deseos y seguir avanzando hacia el no conflicto. El fin de toda guerra es el retorno a una paz parcial, entendiendo que los intereses de uno quedaran sobrepuestos a los del otro sin un afán dominador. El fin de una guerra cuyo objetivo no es la paz supone calificarla de injusta. ${ }^{5}$

\footnotetext{
${ }^{2}$ Immanuel Kant, La paz perpetua (Madrid: Alianza Editorial, 2016), 152.

${ }^{3}$ Tomás García López, "Filosofía de Guerra" [video], Fgbuenotv, 14 h 30 min. 7, 8, 14, 15 y 18 noviembre 2011. http://www.fgbueno.es/act/efo010.htm

${ }^{4}$ El conflicto puede ser constructivo o destructivo, pudiendo así propiciar cambios positivos. Para ello es necesario que se gestionen correctamente. Es importante entender que hacer un diagnóstico correcto del conflicto va a determinar el éxito en la resolución de las distintas problemáticas. Elena Gaviria, "Conflictos y estrategia de solución", en Ángel Gómez, Elena Gaviria Stewart e Itziar Fernández Sedano, Psicología Social (Madrid: Sanz y Torres, 2006), 449-498.

${ }^{5}$ Carl von Clausewitz, De la Guerra (Madrid: La Esfera de los Libros, 2014), 776.
} 


\section{Guerra sin sentido}

El primer error en la GWOT aparece ya en su propia definición al dictaminarse una guerra contra el terrorismo, concepto en el que los implicados difieren. Me permito citar D. Federico Aznar-Fernández Montesinos cuando dice

Quien tiene el poder es el que otorga las definiciones. Existe una definición de acto terrorista pero no una definición de terrorismo como tal. Sin embargo, una definición acertada podría ser: "la utilización mediática de una cierta violencia en beneficio de un proyecto político [...de este modo...] la guerra contra el terrorismo [...] es un martillo declarando la guerra a otro martillo $[\ldots]$.

Una guerra es un choque de voluntades que se resuelven en el campo de batalla, lo que no se resuelve en el campo de batalla son los problemas que no se originan allí, problemas de religión, cuestiones de identidad, credo, etc. Es más no existe un problema militar que sea grave, en el sentido que con una fuerza más o menos razonables se puede resolver. El problema no es ganar la guerra, es ganar la paz ¿Y cómo se gana la paz? La victoria es un asunto demasiado simple para este tipo de cosas, la clave se sitúa en resolver un problema enquistado en la sociedad. La solución es robustecer la sociedad a través del fortalecimiento del Estado y el papel de las fuerzas armadas sería contener esa violencia para que sean ellos los que resuelvan ese tipo de problema reconstruyendo sus estructuras de estado y por consiguiente la sociedad. ${ }^{6}$

Al hilo de esta reflexión resulta evidente que definirla como guerra contra el terrorismo ha sido un grave error por dos motivos: primero, denominarla guerra resulta un absurdo: el fenómeno islamista no se trata de un choque de voluntades que se resuelven en el campo de batalla; en segundo lugar, el terrorismo como tal no puede ser el enemigo ya que terrorismo es un instrumento de guerra y una herramienta política. En todo caso, el enemigo serían los individuos que emplean el terrorismo.

En la misma línea, el ex primer ministro francés Dominique de Villepin añade:

Las intervenciones militares cuando son circunscritas a un objetivo determinado y limitado pueden ser eficaces. Sin embargo, la guerra contra el terrorismo no se puede ganar. Es un fracaso absoluto por qué el terrorismo ${ }^{7}$ es un mano invisible, mutante, cambiante y oportunista. Estamos aquí contrasentido, la GWOT es una guerra sin fin, una guerra que no se puede parar. ${ }^{8}$

\footnotetext{
${ }^{6}$ Pedro Baños, "Visión geopolítica: terrorismo y guerra asimétrica" [video], Tendencias21TV, 16 de octubre de 2015. https://www.youtube.com/watch?v=MHG_5A4VPyE.u.

${ }^{7}$ El ex primer ministro se refiere al terrorismo yihadista global, cuya definición más adecuada la podemos encontrar en Javier Jordán, "Contextualización conceptual, ideológica e histórica del terrorismo global", en Eugenia López-Jacoiste (Coord.), Seguridad, defensa y desarrollo en el contexto internacional actual (Pamplona: EUNSA, 2010).

${ }^{8}$ will, "Villepin: La guerra contra el terrorismo no se puede ganar con las armas de la guerra" [video] France2, el 18 de noviembre de 2015. https://www.youtube.com/watch?v=J0wLKx9Tfwo\&t=2s.
} 
En todo caso, se podrá vencer a las organizaciones terroristas que lo emplean, pero no al terrorismo como instrumento. ${ }^{9}$

Con una mejorada conceptualización y un enfoque distinto, Occidente habría podido centrarse en focalizar sus fuerzas con miras a reforzar las estructuras sociales de estos Estados para que sean ellos mismos quienes den solución al problema, sin obviar las dificultades y complejidades que esto conllevaría.

Calificar la lucha contra el terrorismo como guerra ha originado un problema de base con difícil solución. Además de los conflictos externos que han brotado a tenor de la misma y junto a estos el aumento del terrorismo, en el ámbito interno el carácter globalizador de la respuesta ha originado un incumplimiento del contrato social donde la seguridad Occidental se ha visto deteriorada.

\section{Guerra injusta}

La justicia adquiere pretensión de validez universal al ajustarse a los derechos humanos. La idea genérica universal sobre la justicia existió históricamente en la mayoría de las civilizaciones occidentales y orientales, en culturas primitivas, en el pueblo hebreo del Antiguo Testamento y en el origen de la cultura griega occidental. "Algo es justo cuando su existencia no interfiere el orden al que pertenece; es justo que cada cosa ocupe su lugar en el universo. De tal modo, hay o se produce injusticia cuando una cosa usurpa el lugar de otra, cuando no se confina a ser lo que es o se produce alguna desmesura". ${ }^{10}$

Bajo tales premisas y la desmesura de los actos que se han llevado bajo la justificación de una Guerra contra el Terrorismo resulta conveniente indagar sobre si esta ha sido una guerra justa o, por lo contrario, una guerra injusta.

La doctrina de la guerra justa ha ido evolucionando de modo tal que al estar íntimamente ligada a las características de la comunidad política de cada momento el

\footnotetext{
${ }^{9}$ Entendiendo por terrorismo la definición que ofrece Fernando Reinares al entenderlo como "un conjunto de acciones violentas que generan, en un determinado agregado de población, efectos psíquicos desproporcionados respecto a sus consecuencias materiales y que tiene como fin condicionar las actitudes de dicho colectivo social y orientar sus comportamientos en una determinada dirección".

10 Juan Carlos Tealdi, "Justicia y Derechos Humanos", en Acta Bioeth Diccionario Latinoamericano de Bioética (Santiago: Acta Bioteh, 2010).
} 
concepto es dependiente: del momento histórico, el Estado, el imperio o la polis. La evolución histórica de la guerra difiere en demasía al actual puesto que antiguamente éstas eran contempladas como un mal necesario o un mal menor, siendo justas todas aquellas cuyo fin era alcanzar la paz.

Para poder analizar sobre las guerras del presente es necesario regresar al pasado e investigar sobre lo que podríamos llamar "las doctrinas filosóficas de guerra". Tener en cuenta lo que sucede en la actualidad para conectarlo con el pasado inmediato y así sucesivamente. Por ello estudiar a los autores de mayor relevancia en la materia nos permitirá enmarcar dicha conceptualización para a posteriori poder calificar la GWOT de justa o injusta.

\section{Cicerón}

El político romano afirmaba: "aunque la guerra sea un hecho inevitable, siempre es preferibles la paz" argumentando que "son justas las guerras defensivas, también la guerra movida por el deseo de poder y gloria, e impugna y tacha de injustas aquellas guerras cuyo objetivo es la avaricia". ${ }^{11}$

La GWOT ha sido fruto de una hegemonía americana, un intento de occidentalización y una supremacía de valores. Para Fukuyama la avaricia ha cegado a Estados Unidos a la hora de establecer un liderazgo mundial:

Ofrecemos mercado libre y democracia, dos cosas buenas e importantes que constituyen la base del crecimiento y el orden político. Por lo contrario, ninguna de las dos parece atraer a las poblaciones más pobres que son en definitiva los auténticos electores en esta lucha por el poder e influencia en el mundo. ${ }^{12}$

De este modo, y alejándose del neoconservadurismo, Fukuyama casa su interpretación con las guerras injustas de Cicerón donde la arrogancia avara es causa de injusticia. ${ }^{13}$

\footnotetext{
${ }^{11}$ Marco Cicerón, Sobre los deberes (Madrid: Editorial Alianza: 2015), 296.

12 Francis Fukuyama, "El fin de la hegemonía americana”. El País, 31 de julio de 2008.

${ }^{13}$ Francis Fukuyama, “After Neoconservatism”, The New York Times, 19 de febrero de 2006.
} 


\section{San Agustín}

La teoría del pensador de la Edad Media no ha quedado obsoleta, (cabe destacar que tales argumentaciones fueron afianzadas bajo un contexto cristiano de amor y perdón al ofensor con la defensa de Impero Romano ya en declive.): "Las guerras son justas cuando su fin es el restablecimiento y la obtención de la paz", "No se admiten las injurias como causa de guerra". 14

La Guerra contra el Terror y sus intereses no parecen tener fines pacifistas. Claro ejemplo de ello es la guerra de Irak, decidida con anterioridad al 11 de septiembre y cuyo objetivo era obtener el control de los recursos naturales y garantizar la presencia militar en la zona. Declarar la guerra al terrorismo no ha hecho más que fomentarlo. Si el fin de una guerra justa para San Agustín hubiera sido: "mejorar la seguridad interna", ${ }^{15}$ tal denominación parecería apropiada. Sin embargo, como su fin era la paz y la GWOT no tenía tales pretensiones podemos afirmar que es injusta.

\section{Santo Tomas de Aquino}

Siguiendo a San Agustín y bajo las influencias de la filosofía aristotélicas el filósofo va un paso más allá. Para Santo Tomas las guerras serán justas en la medida que cumplan tres requisitos: ${ }^{16}$

1. "Sólo el príncipe ostenta la competencia necesaria para ordenar un mandato de tal envergadura como es el de hacer la guerra pues es de sus competencias el cuidado y la defensa de los intereses públicos de su comunidad política”. La doctrina unilateralista de Bush no contaba con el apoyo la comunidad política. Europa en su conjunto no compartía la Patriot Act, ni las cárceles de Guantánamo, ni las torturas de Abu Graib, ni la invasión de Irak. Basándose en el

\footnotetext{
${ }^{14}$ Agustín de Hipona, La ciudad de Dios (México: FV Editions, 2015), 730.

15 Javier Jiménez. "Eficacia de la "guerra al terror". Luces y sombras de la seguridad internacional en los albores del siglo XX” Comunicación presentada en las Jornadas de Estudios de Seguridad 2, Madrid, 2010. https://dialnet.unirioja.es/servlet/articulo?codigo=5387140. Así como la mejora de los servicios de inteligencia e información, tanto a niveles nacionales como internacionales".

16 Tomás de Aquino, La suma teológica mínima (Madrid: Tecnos, 2014), 184.
} 
documento "The National Seurity Strategy of the United States of America"17 (septiembre de 2002) el presidente justifica la intervención dando uso de su punto cuarto en el que proclama: "la intervención unilateral cuando sea precisa para salvaguardar los intereses nacionales". Tal unilateralismo arrebata una competencia atribuida al Consejo de Seguridad de la ONU anulando este primer requisito de guerra justa. ${ }^{18}$

2. "Es preciso la existencia de una justa causa, por esta se entiende: la culpa de uno de los beligerantes por lo que la acción bélica solo debe utilizarse para reparar alguna injuria”. Discutible es la existencia de una justa causa. Tzvetan Todorov establece siete razones posibles para la invasión de Irak: ${ }^{19}$

1) La posesión de armas de destrucción masiva.

2) El apoyo al terrorismo de Al Qaeda.

3) El primer paso para la resolución del conflicto árabe-israelí al eliminar el gran obstáculo que constituía Sadam Hussein.

4) Controlar las reservas de petróleo para favorecer a los grandes magnates americanos.

5) Aumentar el prestigio presidencial con vistas a la reelección.

6) Presiones de la industria militar y los militares para la obtención de beneficios.

7) La expansión de la democracia, el argumento neocon según el cual la caída de Sadam Hussein significaría el comienzo de una era de prosperidad para la zona.

3. La intención de los combatientes ha de ser recta: "debe tener como finalidad el bien y la prevalencia de este frente al mal". Los presidentes Bush padre y Clinton lograron en el concierto interno un grado de complicidad porque sus políticas realistas trataron de ser respetuosas con las opiniones de sus aliados y la legalidad internacional siempre que pudieron. Pero, con el presidente Bush hijo en la Casa Blanca todo cambió. El 11 de septiembre le superó y trajo consigo un

\footnotetext{
17 National Security and Defense, “A New National Security Strategy for a New Era”, The White House. http://www.whitehouse.gov/nsc/nss.pdf.

${ }_{18}$ María Paz Andrés, La seguridad comprometida: nuevos desafíos, amenazas y conflictos armados (Madrid: Tecnos, 2008), 36-37.

${ }^{19}$ Tzvetan Todorov, El nuevo desorden mundial (Barcelona: Península, 2003), 19-27.
} 
conjunto de acciones desproporcionadas. Un claro convencimiento de la supremacía de los valores americanos hizo que se lanzará a un combate, la "guerra contra el terror" en el que no contemplo más estrategia que la destrucción del enemigo. Una emotividad canalizada e intereses cuestionables alejarían a la GWOT de este tercer requisito. ${ }^{20}$

\section{Hugo Grocio}

Uno de los padres tanto del derecho internacional como del derecho natural, el jurista sigue la misma línea de los demás autores clásicos al defender la licitud de la guerra en relación al objetivo de la misma, la paz: "Digno es esto de vuestra piedad, digno de esa dignidad, no atacar con las armas el derecho de cualquiera, no alterar los confines antiguos; sino, en la guerra, buscar negociaciones de paz, y no comenzarla sino con este deseo, de terminarla cuanto antes". ${ }^{21}$ El poeta neerlandés matiza que además debe existir un "peligro inminente" y "el uso de la fuerza debe ser proporcional a la amenaza".

La doctrina de Grocio destaca por su humanización: además de existir una causa justa deben respetarse una serie de reglas; aparece el concepto de 'límites', éstos deben ser respetados durante la acción bélica. Por ejemplo, “debe evitarse la destrucción de bienes ajenos a la guerra [...] obras de arte, templos [...] y otros bienes materiales". ${ }^{22}$

Aplicándolo al caso del 11 de septiembre, sus víctimas no son comparables al desastre de una guerra; la respuesta de EE. UU. fue deshumanizada y desproporcional. Otro indicio que refleja el descomedido de la GWOT es que se utilizó el término 'guerra' para combatir un fenómeno que tiene más de actividad criminal que de combate militar.

En segundo lugar, los límites de lo que habla el autor holandés han sido sobrepasados si se analiza el número de civiles fallecidos o bienes materiales destruidos entre tanto caos. ${ }^{23}$

\footnotetext{
${ }^{20}$ Véase nota 14.

${ }^{21}$ Hugo Grocio, Del derecho de la guerra y la paz (Reus: Editorial Reus, 1925), 336.

${ }^{22}$ Ibid., 261.

${ }^{23}$ El informe del Instituto Watson de Asuntos Internacionales y Públicos, de la Universidad de Brown, cifró el número de muertos entre 480000 y 507000 personas, pero aseguró que la cifra probablemente sea más alta en la actualidad. Recogido en: https://www.eluniverso.com/noticias/2018/11/08/nota/7040001/casi-mediomillon-personas-han-muerto-guerra-contra-terrorismo-tras.
} 


\section{Francisco Suarez}

"La guerra no debe ser rechazada, y es acorde con lo dispuesto en el Antiguo testamento. Esto significa que el uso de la fuerza no es intrínsecamente malo". ${ }^{24}$ El teólogo defiende que para que una guerra sea justa debe cumplir los tres requisitos que establece Santo Tomas. Además, se atreve a discernir entre el bellum defensivum y el bellum agressivum. La guerra defensiva es justa en la medida que su justificación va ligada a la legítima defensa. En cuanto a la ofensiva, considera que es difícil encontrar en ella una causa justa. Aun así, entiende que será legitima siempre que su fin sea restablecer la paz o evitar una injusticia.

Luis M. Hinojosa Martínez explica que no basta con el apoyo del Consejo de Seguridad para invocar la legítima defensa.

Esta figura se encuentra regulada en el art. 51 de la Carta de las Naciones Unidas que tal y como ha sido interpretado por el Tribunal Internacional de Justicia (TIJ) establece una serie de condiciones materiales y procedimientos para su válida invocación: (a) debe haberse producido previamente un ataque armado, (b) la respuesta debe ser necesaria y proporcionada y (c) se debe informar inmediatamente al Consejo de Seguridad para que éste adopte las decisiones que estimen oportunas. ${ }^{25}$

En cuanto a las condiciones basta con acudir al apartado b) para contemplar como la proporcionalidad fue vulnerada argumentado que "la amplitud de la actuación militar sobrepasaría con creces los permitido por este principio".

Si no se trata de una guerra defensiva no queda otro calificativo que el de guerra ofensiva. Claro ejemplo es el nombre que la GWOT adoptó; al denominar guerra contra el terrorismo abrió un amplio abanico de posibilidades eliminando la necesidad de establecer un enemigo concreto. Habría sido una guerra defensiva si la guerra se hubiera declarado contra Al-Qaeda, Bin Laden o los Talibanes. Sin embargo, generalizar el concepto ha permitido a Estados Unidos ampliar un campo de juego con miras a una occidentalización forzada. El Doctor Eximius no duraría en declarar la GWOT como bellum agressivum, sin causa justa lo que derivaría en una guerra injusta en toda regla.

\footnotetext{
${ }^{24}$ Luciano Pereña, Teoría de la Guerra en Francisco Suarez (Madrid: Instituto Francisco de Vitoria, 1954), 333.

${ }^{25}$ Luis Miguel Hijonosa Martínez, "Irak y Afganistán: una comparación desde el derecho internacional”, Real Instituto Elcano, núm. 10 (enero, 2008): 3-4.
} 


\section{Francisco de Vitoria}

El fraile da seguimiento a la tesis tomatista de los tres requisitos indispensables de guerra justa. Sin embargo, observó algunas lagunas en éstos dado que era muy sencillo recaer en un error de buena fe. Observando el conflicto bélico desde dentro resulta más probable que las partes beligerantes opinen subjetivamente que luchaban por una causa justa. ${ }^{26}$

Pasados seis meses del trágico 11 de septiembre, se publicó una "Carta desde América", por el Instituto de valores americanos y firmado por intelectuales estadounidenses entre los que se encontraban Samuel Huntington y Francis Fukuyama. En ésta los firmantes consideraron que la guerra contra el terrorismo era una guerra justa. La justificación de tal afirmación se basó en que

esos valores son universales; la democracia como expresión política más clara y trascendente de la dignidad humana; el convencimiento de que existen verdades con carácter universal, el conocimiento de la verdad es imperfecto por lo que es indispensable la tolerancia para salvar discrepancias; y la libertad de pensamiento y religiosa. ${ }^{27}$

Para Francisco de Victoria excusar la GWOT bajo tales razonamientos morales no habría sido más que una laguna que debilitaría el sistema de los tres requisitos de Santo Tomas. Del mismo que hizo Occidente, el terrorismo yihadista podría hacer la Yihad bajo una opinión subjetiva dando pie una guerra santa.

\section{La GTWO: pseudoguerra, occidentalización enmascarada}

En primer lugar, conforme a lo argumentado en el punto anterior, la GWOT sería una pseudoguerra y no una guerra como tal. Los actos conllevan definiciones y calificar algo con su propia negación resulta insuficiente. Entonces ¿En qué consiste la GWOT?

La pseudoguerra contra el terror es un proceso de paz tensa posterior a la Guerra Fría, mediante el cual Occidente, liderado por Estados Unidos, ha intentado mantener su

\footnotetext{
${ }^{26}$ Victoria García, La teoría de la guerra justa en Francisco de Vitoria [tesis final de grado], Universidad de Valladolid, España, 2019,59-60.

27 Adrián Liman, El caos que viene (Madrid: Editorial Popular, 202), 109-124.
} 
hegemonía a través de guerras ficticias y sin sentido. Bajo un ideario de guerra perpetua, la primera potencia mundial pretende preservar y ampliar la democratización del globo. Para mantener dicho orden mundial el control de la civilización Oriental resulta indispensable y la GWOT es un mero instrumento al servicio de un proceso de occidentalización que apenas acabaría de comenzar y que por el momento está fallando.

\section{Pseudoguerra de intromisión: cambio estratégico}

La GWOT ha servido de trampolín para actuar bajo intereses de diferente rango y mediante una estrategia intervencionista respaldada por las resoluciones 1970 y 1973 y siguientes del Consejo de Seguridad de la ONU que choca con el principio de no intervención. Además, esta intervención lejos de resultar fructífera se ha basado en una permisibilidad y un garantismo que ha dado pie a que se origine una intromisión inactiva fomentado el famoso choque de civilizaciones, expuesto por Huntington.

Este conflicto entre civilizaciones ha incrementado el sentimiento radical islámico dando pie a que las organizaciones terroristas se conviertan en pseudo-Estados. Occidente pretende la imposición de un modelo político y de Estado contrario a los valores y principios del mundo islámico. La GWOT ha resultado errónea en el momento que focalizó sus actuaciones hacia el exterior; ampliar su campo de actuación la ha debilitado y ha dado motivos al enemigo para combatirnos en nuestro propio territorio (desde entonces en el punto de mira). La respuesta lejos de ser eficaz ha propiciado que los Estados implicados actúen aturdidos tras sus fronteras olvidando el contrato social y obligación de proteger a sus ciudadanos. La respuesta estaba en casa y fuimos a buscarla a la del vecino. ${ }^{28}$

Lawrence Freedman, un prestigioso historiador militar británico, ha publicado recientemente The Future of War $^{29}$ donde evidencia como la GWOT es una guerra sin victoria. En primer lugar, se trata de una guerra contra principios establecidos por quienes la llevan a cabo, algo ilógico. El principio de no intervención fue vulnerado en su totalidad

\footnotetext{
${ }^{28}$ Francisco Berenguer, "La respuesta político-diplomática en AA. VV., Estrategias para derrotar al Dáesh y la reestabilización regional", Cuadernos de Estrategia Instituto Español de Estudios Estratégicos, (noviembre, 2016): 21-42.

${ }^{29}$ Lawrence Freedman, The Future of War (New York: PublicAffairs, 2017), 400.
} 
cuando esta oleada intervencionista — aplaudida en algunos casos como Kuwait o BosniaHerzegovina - cayó en una ineficacia absoluta con las intervenciones en Irak o Libia. ${ }^{30}$ Para Federico Aznar Montesinos Fernández "todo se puede defender en derecho, todo depende de las políticas de poder" ${ }^{\prime 3}$ y esto es lo que sucedió, para lograr sus objetivos se justificó lo injustificable. Igual que ocurre en metafísica, una desalineación entre nuestro ser y nuestras acciones conlleva resultados fallidos. Cuando lo que decimos y hacemos no va en concordancia el fracaso está asegurado.

El 11 de septiembre su consecuente declaración de la GWOT no fueron más que un cambio estratégico sobre unas propuestas decididas previamente. ${ }^{32}$ La única novedad que presenta tal declaración es la invasión de Afganistán cuya intervención se consideraría positiva y acorde a la concepción de guerra justa. Sin embargo, la guerra contra el terror fue una oportunidad bien aprovechada, dividir ideológicamente para obligar a adoptar una posición nosotros o ellos. La estrategia contra el imperio del mal se transformaría en la de contra el eje del mal, ${ }^{33}$ conformado por Irak, Irán y Corea. ${ }^{34}$

\section{Pseudoguerra ajena: democracia balística}

La GWOT no ha sido una respuesta bélica ante el terrorismo yihadista sino un intento de occidentalización mediante el cual se ha pretendido que las sociedades orientales adoptarán un modelo político a medida. Tal imposición ha resultado fallida al ignorándose ciegamente los tres grandes problemas internos del islam: ${ }^{35}$

1. Mahoma muere sin señalar sucesor, de forma que el poder político y religioso quedan unidos para siempre.

2. El profeta falleció sin constituir un clero, de modo que no existe una autoridad suficiente para interpretar sus escrituras.

30 Antonio Rubio, “Guerras sin victorias", Real Instituto Elcano, Firmas invitadas (Política global) (noviembre, 2018): 5.

${ }^{31}$ Sergio Fernández, Vivir con abundancia (Barcelona: Plataforma Editorial, 2015), 240.

${ }^{32}$ Las sanciones sobre Irak comenzarían en 1991 y duraría hasta el 2003.

${ }^{33}$ La frase Evil empire (Imperio maligno o malvado) fue acuñada por el presidente estadounidense Ronald Reagan en 1983 para referirse específicamente a la entonces Unión Soviética de Yuri Andrópov.

${ }^{34}$ Oliver Roy, Después del 11 de septiembre (Barcelona: Bellaterra, 2007), 17-18.

35 José Javier Esparza, La historia de la Yihad (Madrid: La Esfera de los Libros, 2015), 400. 
3. No se matizó que "El combate por la fe" (Yihad) y la aniquilación era meramente espiritual y no física.

Así pues, el islam supone un problema en sí mismo, fuera de nuestro alcance y ante el cual deberíamos plantearnos la posibilidad que sea el mundo islámico el que recorra su camino por sí solo. La GWOT (intento de occidentalización) es considerada como terrorismo ofensivo o terrorismo de la uniformización, semejantes al de Hitler y Stalin. La guerra contra el terror es conocida también como una democracia balística a través de la cual Occidente pretende imponer un sistema de valores, político e institucional propio.

Como defienden los especialistas Federico Aznar y Jorge Verstrynge: "imponer valores a alguien o instituciones que surgen en una cultura sobre otra cultura distinta, hace que esas instituciones no funcionan [...] Jamás habrá una revolución que triunfe en un país islámico que venga del exterior, claro ejemplo de ello es lo sucedido en Afganistán". ${ }^{36}$

El cosmopolitismo impulsado por Karl Marx y Friedrich Engels y la idea sustentada sobre "Las demarcaciones y los antagonismos nacionales entre los pueblos desaparecen cada vez más" ${ }^{37}$ ha originado que Occidente promulgue valores y principios éticos como es la "integración", incompatibles con el credo islámico. Se trata así de un prejuicio occidental fundado en la idea de que el fin es la convergencia entre todos cuando se tratan de realidades culturales totalmente distintas. Aceptar las diferencias supondría una valoración positiva enfocada a futuras actuaciones. El éxito radica en entender que las intervenciones en civilizaciones orientales están encaminadas al fracaso debido al choque cultural, que cualquier imposición ajena será rechazada y que una posible occidentalización de Oriente sólo será posible mediante una iniciativa propia de la comunidad islámica.

\section{Pseudoguerra de civilizaciones: nuevo orden mundial}

Después de cada gran conflicto nace la esperanza de que de los desastres de la guerra nacerá una "paz duradera". Esta esperanza trae consigue una modificación del orden

\footnotetext{
${ }^{36}$ Véase nota 5.

${ }^{37}$ Wenceslao Roces, El manifiesto comunista, introducción histórica. (Madrid: Cenit, 1932), 1-18.
} 
establecido o una recuperación del anterior. Al finalizar la primera guerra mundial el presidente de Estados Unidos fue el primero en utilizar el concepto de 'nuevo orden mundial' y la comunidad internacional se orientó hacia su construcción creando la Sociedad de Naciones.

La Segunda Guerra Mundial demostró como éste fracasó estrepitosamente y de ella se confeccionó la ONU, pese a presentar similares pretensiones. Al finalizar la Segunda Guerra Mundial el mundo se dividió en dos ejes; dos grandes superpotencias enfrentadas económica, social y políticamente. Transcurrida la Guerra Fría, Estados Unidos salió victorioso y con la preeminencia que lo caracteriza empleó el intervencionismo frente a terceros países. Como consecuencia de tal intervencionismo surgió una nueva modalidad de terrorismo, un terrorismo yihadista al que se le declararía la guerra. ${ }^{38}$

El nuevo orden mundial adquiere una connotación similar al de la "paz duradera" de Kant. ${ }^{39}$ Como comentábamos, las guerras del presente exigen paces tensas y paces parciales, como la que se desarrolló durante la Guerra fría. De este modo, un nuevo orden mundial no sería tanto la búsqueda de una paz duradera, si no un cambio de paradigma bélico.

Dicha consideración liga a la perfección con la filosofía política straussiana, que cree más en la 'perpetua guerra' que en la 'paz perpetua'. El influyente Leo Strauss defiende tres tesis que vendrían a justificar las políticas occidentales: primero sostiene que para hacer seguro el mundo para las democracias occidentales es necesario democratizar el globo; segundo, es necesario acabar con las democracias laicas a favor de democracias ligadas a las confesiones religiosas para crear una verdadera moral pública. Tercero, para cohesionar a la nación es necesario exacerbar el patriotismo. De este modo las democracias requerirían un enemigo real o ficticio para ser cohesionadas. Ayer era Moscú; hoy, el terrorismo. ${ }^{40}$

Así el straussianismo aparece como una extrapolación filosófica política de la funcionalidad criminal que postuló exitosamente Emilie Durkheim, ${ }^{41}$ quien considera el crimen como un mal necesario para que se desarrollen todas las condiciones fundamentales

\footnotetext{
${ }^{38}$ Oscar Domenech, "En que consiste el nuevo orden mundial” [video], UnProfesor, 3 de agosto de 2019. https://www.youtube.com/watch? $\mathrm{v}=\mathrm{R}$ 3UiC5e-w8.

${ }^{39}$ Immanuel Kant, La paz duradera (Barcelona: Tecnos, 2013), 112.

${ }^{40}$ Eduardo Pizarro, "Usa y el Nuevo Orden Mundial”, El Tiempo, 26 de mayo de 2003.

41 Omar Huertas, "Durkheim: la perspectiva funcionalista del delito en la criminología", Revista Criminalidad, núm. 2 (diciembre, 2009):1-13.
} 
de toda vida social. ${ }^{42}$ De la misma manera, Strauss concibió la guerra como un instrumento indispensable para lograr una democratización global. Tales inferencias nos llevan a contemplar la GWOT como un continuo, una necesidad social que surge al finalizar Guerra Fría. El problema radica en que esta pseudoguerra no es una guerra contra el terrorismo sino frente a un conjunto de sistemas que amenazarían la hegemonía de Estados Unidos y el sistema democrático occidental como primera potencia mundial. Declararla cómo guerra contra el terror fue un hecho oportunista con la finalidad de sumar adeptos a la causa.

Este oportunismo trajo consigo que un gran número de colaboradores de la comunidad internacional se unieran a ella influenciada por la tríada de Hegel. ${ }^{43} \mathrm{El}$ afán democratizador previo al 11 de septiembre originó una respuesta indirectamente buscada que causaría 2992 fallecidos, cifra suficiente para promover la ya iniciada guerra por la occidentalización o como se le denominó posteriormente, la GWOT. ${ }^{44}$ La estrategia hegeliana se fundamentó en lo que se conoce como la tríada dialéctica: tesis, antítesis y síntesis: "Trasladado al campo político se traduce en crear un problema y venir yo a poner la solución de ese problema para que la opinión pública apruebe la agenda oculta. Dicho en otras palabras, crear un enemigo". ${ }^{45}$

Las teorías de Strauss y Hegel no llevan a aceptar el choque de las civilizaciones. $^{46}$ Como predijo Huntington, el mundo de las civilizaciones desarrollaría su propia estructura. Finalizada la Guerra Fría los conflictos no serían ni económicos ni políticos, sino culturales; las nuevas guerras enfrentarían a civilizaciones antagónicas donde la occidental deberá defender su hegemonía frente el resto. Así, la guerra contra el terror ha pretendido una occidentalización que no se está produciendo. Para lo que a occidente supone una instauración de democracias liberales a través del universalismo ${ }^{47}$ para el resto del mundo resulta ser un imperialismo. Promover los valores democráticos occidentales y los derechos humanos se considera una intromisión en asuntos internos cuyo único fin es su expansión económica.

\footnotetext{
${ }^{42}$ Emilie Durkheim, Las reglas del método sociológico (Barcelona: Libros de Pon, 1983), 128.

${ }^{43}$ Sergio Casado, "Transformando sociedades. La triada de Hegel", Revista Libertalia (7 de junio de 2017): 5.

${ }^{44}$ Norbert Elías, La sociedad de los individuos (Barcelona: Editorial Península, 1990), 134. "Nacido de planes, pero no planeado. Movido por fines, pero sin un fin".

45 Alberto Villasana, "Nuevo orden mundial" [video] Conclusión TV, 1 de febrero de 2017. https://www.youtube.com/watch?v=ZmhGbB_KT0U.

46 Samuel P. Huntigton, "The Clash of Civilizations", Foreign Affaris, núm. 32 (verano, 1993): 28.

${ }^{47}$ Movimiento político que propugna la unidad de todos los pueblos o los estados y la eliminación de los obstáculos que impiden su plena comunicación y relación.
} 
De este modo, las pretensiones de universalización generan conflictividad debido a que las relaciones internacionales se encuentran fuertemente vinculadas a la cultura y los valores. Los esfuerzos por una libertad de fronteras, la globalización y universalismo occidental ha generado una crisis de identidad que ha tenido como repuesta un mayor sentimiento de arraigo civilizacional. La GWOT, lejos de acabar con el terrorismo, ha fomentado que este mismo sea una herramienta ideal para defender su identidad. "Sabemos quiénes somos, sólo cuando sabemos quiénes no somos y con frecuencia sólo cuando sabemos contra quienes estamos". ${ }^{48}$ Fukuyama, a pesar de tener una posición contraria a Huntington, coincide en que "uno no disemina la democracia invadiendo países". 49

El intervencionismo como respuesta ha supuesto una legitimación y reforzamiento de la tesis yihadista. La pretensión universalista de Occidente tras el 11 de septiembre supone una paradoja democrática cuando los derechos fundamentales propios ${ }^{50}$ (tan garantistas) producen ventajas a las militancias yihadistas. Es por ello que la guerra contra el terror falló: no se tuvo en cuenta un nivel macro capaz de iniciar o acelerar los procesos de radicalización. ${ }^{51}$ Más allá de responder reactivamente a los atentados producidos el 11 de septiembre, Occidente desencadenó una guerra contra el terror iniciada ya con anterioridad. Un afán mayor al de la propia represalia pretendió utilizar los acontecimientos como fuerza motriz hacia una occidentalización imposible.

\section{Conclusiones}

La GWOT ha fracasado: la occidentalización de Medio Oriente —iniciada ya hace más de 30 años con la globalización occidentalizada y el liderazgo selectivo de Clinton, seguida por el imperialismo de Bush hijo y el internacionalismo idealista de Barack Obama - ha llegado a su fin. Tres décadas después se pretende hacer grande a Estados Unidos de nuevo (Make America Great Again), pero solamente para en el ámbito interno. El nuevo orden mundial ha dado un

48 Samuel P. Huntington, The Clash of Civilizations and Remarking of World Order (Barcelona.: Ediciones Paidós, 1997), 367.

${ }^{49}$ Francis Fukuyama, El fin de la historia. Y otros ensayos (Madrid: Alianza Editorial, 2015), 168.

${ }^{50}$ En otros, la libertad de expresión y de asociación, la presunción de inocencia, la necesidad de contar con autorización judicial para interceptar las comunicaciones.

51 Javier Jordán, "Procesos de radicalización yihadista en España. Análisis sociopolítico en tres niveles", Revista Psicología Social, 2 (marzo, 2009): 197-216. 
giro de ciento ochenta grados donde el concepto de Estado-nación se superpone sobre el globalismo. La cumbre de Davos dejó de manifiesto en abanderamiento nacionalista y proteccionista de Donald Trump. El nuevo orden internacional que asumió Estados Unidos para consumo de bienes propios, contrario a toda una historia intervencionista.

Este cambio de estrategia se originó en gran medida en el momento que Estados Unidos ha evidenciado como imposible este intento de occidentalización de Medio Oriente camuflado bajo la conceptualización de GWOT. Tanto la Guerra Fría como la GWOT han sido más que dos paces parciales dentro de una guerra perpetua que resulta tan necesaria para que una primera potencia mundial como es Estados Unidos mantenga su liderazgo.

El nuevo paradigma daría por finiquitada la GWOT como intento de occidentalización fracasado. La guerra contra el terrorismo es una guerra sin fin. El terrorismo existió, existe y existirá, pero al parecer ya no será utilizado como trampolín para otros intereses. El mundo islámico ha sido el mayor perjudicado, el terrorismo ha aumentado y la inestabilidad de los Estados se ha visto todavía más mermada. Estados Unidos movió el avispero y ahora se coloca el traje de apicultor.

Si Joseph Biden, nuevo presidente de Estados Unidos, adopta una política exterior como la mantenida por Donald Trump, el liderazgo mundial continuará, pero desde una nueva perspectiva contraria al multilateralismo pretendido años atrás. Encerrados en sí mismos, aparecerán nuevas guerras o paces parciales donde nuevas formas de terrorismo tomarán protagonismo; el ciberterrorismo y terrorismo financiero se llevarán un gran número de papeletas, siendo los nuevos aliados de un terrorismo convencional eterno.

El choque de civilizaciones proseguirá, las diferencias culturales se incrementarán y la conflictividad se centrará en el ámbito económico. La cultura es inconquistable en gran medida mientras que la economía no. Esperemos que no se declare la guerra al terrorismo financiero o algo similar y se opte por una denominación correcta que permita que la próxima guerra tenga un fin alcanzable y evitar así desastres como el incremento del terrorismo que ha supuesto la tan ambiciosas declaración de la GWOT. La perpetua guerra de Occidente, liderada por Estados Unidos, mantendrá su hegemonía mientras tenga la capacidad de controlar estas paces parciales. Por el momento, la GWOT ha sido una batallada perdida.

¿Cuál será la próxima guerra hacia la occidentalización?

Continuará... 


\section{Bibliografía}

Andrés, María Paz. La seguridad comprometida: Nuevos desafíos, amenazas y conflictos armados. Madrid: Tecnos, 2008.

Aquino, Tomás. La suma teológica mínima. Madrid: Tecnos, 2014.

Aquino, Tomás. Suma de teología. Madrid: Biblioteca de autores cristianos, 1993.

Baños, Pedro. "Visión Geopolítica: Terrorismo y Guerra Asimétrica” [video] Tendencias21TV, 16 de octubre de 2015. https://www.youtube.com/watch?v=MHG_5A4VPyE.

Berenguer, Francisco, "La respuesta político-diplomática en AA. VV., Estrategias para derrotar al Dáesh y la reestabilización regional". Cuadernos de Estrategia Instituto Español de Estudios Estratégicos (noviembre, 2016): 21-42.

Casado, Sergio. "Transformando sociedades. La triada de Hegel”. Revista Libertalia (7 de junio de 2017): 5 .

Cicerón, Marco. Sobre los deberes. Madrid: Alianza Editorial, 2015.

Clausewitz, Carl. De la Guerra. Madrid: La Esfera de los Libros, 2014.

Domenech, Oscar. "En que consiste el nuevo orden mundial" [video]. UnProfesor, 3 de agosto de 2019. https://www.youtube.com/watch?v=R_3UiC5e-w8.

Durkheim, Emilie. Las reglas del método sociológico. Barcelona: Libros de Pon, 1983.

Gaviria, Elena. “Conflictos y estratégia de solución”, en Ángel Gómez Jiménez, Elena Gaviria e Itziar Fernández Sedano (Coords.), Psicología Social, 449-498. Madrid: Sanz y Torres, 2006.

Elías, Norbert. La sociedad de los individuos. Barcelona: Editorial Península, 1990.

Esparza, José. La historia de la Yihad. Madrid: La Esfera de los Libros, 2015.

Fernández, Sergio. Vivir con abundancia. Barcelona: Plataforma Editorial, 2015.

Freedman, Lawrence. The Future of War. New York: PublicAffairs, 2017.

Fukuyama, Francis. "El fin de la hegemonía americana”. El País, 31 de julio de 2008.

Fukuyama, Francis. "After Neoconservatism”. The New York Times, 19 de febrero de 2006.

Fukuyama, Francis. El fin de la historia. Y otros ensayos. Madrid: Alianza Editorial, 2015.

García, Tomás. "Filosofía de Guerra" [video]. Fgbuenotv, 7, 8, 14, 15 y 18 noviembre, 2011. http://www.fgbueno.es/act/efo010.htm. 
García, Victoria. "La teoría de la guerra justa en Francisco de Vitória" [Tesis final de grado], Universidad de Valladolid, España, 2019.

Grocio, Hugo. Del derecho de la guerra y la paz. Reus: Editorial Reus, 1925.

Huertas, Omar. "Durkheim: la perspectiva funcionalista del delito en la criminología". Revista Criminalidad, núm. 2 (diciembre, 2009):1-13.

Hijonosa, Luís. "Irak y Afganistán: una comparación desde el derecho internacional”, Real Instituto El Cano, núm. 10 (enero, 2008): 3-4.

Hipona, Agustín. La ciudad de Dios. México: FV Editions, 2015.

Huntington, Samuel. "The Clash of Civilizations", Foreign Affaris, núm. 32 (verano, 1993): 28.

Huntington, Samuel P. El choque de civilizaciones y la reconfiguración del orden mundial. Barcelona: Ediciones Paidós, 1997.

Jiménez, Javier. "Eficacia de la 'guerra al terror'. Luces y sombras de la seguridad internacional en los albores del siglo XX”. Presentación en las Jornadas de Estudios de Seguridad $2^{\circ}$, Madrid, España, 2010. https://dialnet.unirioja.es/servlet/articulo?codigo $=5387140$.

Jordán, Javier. "Conceptualización conceptual, ideológica e histórica del terrorismo global”. Grupo de Estudios de Seguridad Internacional (GESI) (diciembre, 2010): 137-167.

Jordán, Javier. "Procesos de radicalización yihadista en España. Análisis sociopolítico en tres niveles”. Revista Psicología Social, 2 (marzo, 2009): 197-216.

Kant, Immanuel. La paz duradera. Barcelona: Tecnos, 2013.

Kant, Immanuel. La paz perpetua. Madrid: Alianza Editorial, 2016.

Ley Natural, http://www.leynatural.es/2013/01/11/que-significa-la-veracidad/\#: :text=La\%20v racidad $\% 2 \mathrm{C} \% 20 \mathrm{seg} \% \mathrm{C} 3 \% \mathrm{BAn} \% 20 \mathrm{Jean} \% 20 \mathrm{Paul}$,pero\%20sus\%20presupuestos\%20so n\%20distintos.

Liman, Adrián. El caos que viene. Madrid: Editorial Popular.

National Security and Defense. "A New National Security Strategy for a New Era". The White House. http://www.whitehouse.gov/nsc/nss.pdf.

Pereña, Luciano, Teoría de la Guerra en Francisco Suarez. Madrid: Instituto Francisco de Vitoria, 1954. 
Pérez, Cristóbal. "La Guerra global contra el terrorismo (GWOT)”. Boletín de información, núm. 324 (2012).

Pizarro, Eduardo. "Usa y el Nuevo Orden Mundial”. El Tiempo, 26 de mayo de 2003.

Roces, Wenceslao. El Manifiesto Comunista, Introducción histórica. Madrid: Cenit, 1932.

Roy, Oliver. Después del 11 de septiembre. Barcelona: Bellaterra, 2007.

Rubio, Antonio. "Guerras sin victorias". Real Instituto Elcano, Firmas invitadas (Política global) (noviembre, 2018): 5.

Sartre, Jean-Paul. Verdad y Existencia. Barcelona: Ediciones Paidós, 1996.

Tealdi, Juan Carlos. "Justicia y Derechos Humanos". Acta Bioeth Diccionario Latinoamericano de Bioética. Santiago: Acta Bioteh, 2010.

Todorov, Tzvetan. El nuevo desorden mundial. Barcelona: Península, 2003.

Villasana, Alberto. "Nuevo orden mundial". [video] Conclusión TV, 1 de febrero de 2017. https://www.youtube.com/watch?v=ZmhGbB_KT0U.

Will. "Villepin: La guerra contra el terrorismo no se puede ganar con las armas de la guerra" [video]. France2, 18 de noviembre de 2015. https://www.youtube.com/watch?v=J0wLKx9Tfwo\&t=2s. 